\title{
Two-dimensional model for ferromagnetic martensites
}

\author{
S. Sreekala ${ }^{1}$ and G. Ananthakrishna ${ }^{1,2, *}$ \\ ${ }^{1}$ Materials Research Centre, Indian Institute of Science, Bangalore-560012, India \\ ${ }^{2}$ Centre for Condensed Matter Theory, Indian Institute of Science, Bangalore-560012, India \\ (Received 28 February 2005; revised manuscript received 11 August 2005; published 4 October 2005)
}

\begin{abstract}
We consider a recently introduced two-dimensional square-to-rectangle martensite model that explains several unusual features of martensites to study ferromagnetic martensites. The strain order parameter is coupled to the magnetic order parameter through a four-state clock model. Studies are carried out for several combinations of the ordering of the Curie temperatures of the austenite and martensite phases and, the martensite transformation temperature. We find that the orientation of the magnetic ordering, which generally points along the short axis of the rectangular variant, changes as one crosses the twin or the martensite-austenite interface. The model shows the possibility of a subtle interplay between the growth of strain and magnetic order parameters as the temperature is decreased. In some cases, this leads to qualitatively different magnetization curves from those predicted by earlier mean-field models. Further, we find that strain morphology can be substantially altered by the magnetic order. We have also studied the dynamic hysteresis behavior. The corresponding dissipation during the forward and reverse cycles has features similar to the Barkhausen's noise.
\end{abstract}

DOI: 10.1103/PhysRevB.72.134403

PACS number(s): 75.30.Gw, 75.60.-d, 81.30.Kf

\section{INTRODUCTION}

Ferromagnetic shape memory alloys (FSMAs) like $\mathrm{Ni}_{2} \mathrm{MnGa}$ and $\mathrm{Fe}_{7} \mathrm{Pd}_{3}$ undergo a first-order martensitic transformation on cooling and are also ferromagnetic materials. During the martensitic transformation, the higher symmetry parent phase undergoes a spontaneous deformation to a lower symmetry phase, for instance, from a cubic parent phase to a tetragonal martensitic phase as for the case of $\mathrm{Ni}_{2} \mathrm{MnGa}$. In Ni-Mn-Ga Heusler alloys, the short crystallographic axis is always the easy direction of magnetization. ${ }^{1-4}$ This feature coupled with twinning in the martensitic phase also implies that neighboring variants have a nearly perpendicular easy axis of magnetization. Thus the application of a magnetic field can induce a bias in favor of one of the variants. Moreover, some of these materials have a high degree of magnetic anisotropy. For example, in Ni-Mn-Ga martensites, the magnetic anisotropy in the martensitic phase is $\left(10^{3} \mathrm{~J} / \mathrm{m}^{3}\right)$ two orders greater than the parent austenite phase. ${ }^{5}$ Due to the large anisotropy in such systems, magnetization stays glued to the easy axis of magnetization. Thus in $\mathrm{Ni}_{2} \mathrm{MnGa}$ very large strains can be induced by the redistribution of twin variants caused by the application of even modest strengths of magnetic field. Ullakko et al. ${ }^{6}$ suggested that the magnetic-field induced strain observed in the martensite phase arises from the twin boundary motion rather than magnetostriction. ${ }^{7,8}$ Later much higher strains $\sim 6-7 \%$ have been reported in FSMAs. ${ }^{6,9-12}$ For this reason, FSMAs are good candidates for actuator applications.

A large volume of experimental work has been devoted to study various physical properties characterizing the magnetoelastic transition ${ }^{13-19}$ including their dependence on alloying composition, as the latter affects the transition temperatures. ${ }^{20,21}$ In view of their technological importance, several models have been proposed to explain the large reversible strains. ${ }^{9,10,22,23}$ One feature of FSMA that leads to interesting consequences is the possibility of different Curie temperatures for the parent and the product phases in addition to the martensite transformation temperature. Further, in principle the Curie temperatures could be higher or lower than the martensite transformation temperature. This leads to six possible orderings of the martensite transformation temperature and the Curie temperatures of the two phases. In view of this, there have been studies to obtain the magnetic phase diagrams for different sequences of ordering of the martensite transformation temperature and the Curie temperatures of the two phases using mean-field models. ${ }^{20,21,24-27}$ There have been few attempts ${ }^{22}$ to study the changes in the magnetic order across the variants of the martensites, and also across the martensite-austenite interfaces.

The purpose of the present paper is twofold. First, to study the orientational changes of the magnetic ordering occurring in the martensite variants and in the parent phase for various possible orderings of the Curie temperatures relative to the martensite start temperature. Second, to propose a model that deals with the martensite transformation and the magnetic transition on a similar footing with the aim of capturing the mutual influence of the two order parameters (OPs). This exercise will be carried out within the context of a recently introduced model for square-to-rectangle martensite transformation. This model has been shown to capture $^{28-30}$ several unusual properties associated with athermal martensites such as the power-law statistics of the acoustic emission (AE) signals, the correlated nature of AE signals when the system is cycled in a small range of temperatures and the associated shape memory effect, and the precursors effect. $^{31-35}$ The model is coupled to the magnetic order parameter through a four-state clock model. The clock model is used as a continuous spin system in two dimensions do not support long-range order. While the strain order parameter is governed by a Bales and Gooding type of equation, ${ }^{36}$ the development of magnetic order is carried out using the Monte Carlo method. We find that the magnetic order parameter which is generally oriented along the short axis of the rectangular variant changes as one crosses the martensitic 
twin interface or martensite-austenite interface. We also find that the magnetic order parameter influences the strain and vice versa substantially. We also study the "noise" associated with magnetic hysteresis ${ }^{37-39}$ that is similar to Barkhausen noise.

\section{STRUCTURAL AND MAGNETIC TRANSFORMATIONS}

Let $T_{M s}$ and $T_{M f}$ be the martensite start and finish temperatures, respectively. Defining $T_{c}^{A}$ and $T_{c}^{M}$ to be the Curie temperatures of austenite and martensite phases, we have six different possible ways of ordering of the above temperatures. They are
(a) $T_{c}^{M} \leqslant T_{M s} \leqslant T_{c}^{A}$
(b) $T_{M s} \leqslant T_{c}^{M} \leqslant T_{c}^{A}$,
(c) $T_{M s} \leqslant T_{c}^{A} \leqslant T_{c}^{M}$,
(d) $T_{c}^{M} \leqslant T_{c}^{A} \leqslant T_{M s}$,
(e) $T_{c}^{A} \leqslant T_{M s} \leqslant T_{c}^{M}$,
(f) $T_{c}^{A} \leqslant T_{c}^{M} \leqslant T_{M s}$.

The first four cases are situations where the austenite phase is the first to order ferromagnetically. For the cases (e) and (f), the onset of the ferromagnetic temperature is determined by $T_{M s}$ and $T_{c}^{M}$, respectively. Condition $a$ is more interesting because on lowering the temperature, the para- to ferromagnetic transition takes place in the austenite phase followed by a martensitic transformation which becomes magnetic only on further lowering the temperature.

\section{MODEL}

The total free energy of a ferromagnetic martensite has contributions arising from the elastic energy $F_{e}(\epsilon)$, magnetic energy $F_{m}(\theta)$, and magnetoelastic coupling $F_{m e}(\epsilon, \theta)$ where $\epsilon$ is the order parameter for ferroelastic transformation and $\theta$ is the order parameter for the para- to ferromagnetic transition.

\section{A. Elastic free energy}

Here we adopt a recently introduced model for square-torectangle martensite in two dimensions. While the elastic free energy has contributions arising from all the three strain components, namely the bulk dilational, deviatoric, and shear strains, we have used only the deviatoric strain as the principal order parameter. ${ }^{28-30}$ The effect of the other two strain components are included only in an indirect way (see below). The strain order parameter $\epsilon(\vec{r})$ is defined as ${ }^{28-30}$

$$
\epsilon(\vec{r})=\left(\frac{\partial u_{x}(\vec{r})}{\partial x}-\frac{\partial u_{y}(\vec{r})}{\partial y}\right) / \sqrt{2}=\epsilon_{x}(\vec{r})-\epsilon_{y}(\vec{r}),
$$

where $u_{x}$ and $u_{y}$ are, respectively, the displacement fields in the $x$ and $y$ directions.

The elastic free energy $F_{e}$ is the sum of the local freeenergy functional $F_{L}(\epsilon(\vec{r}))$ and a nonlocal long-range term $F_{L R}(\epsilon(\vec{r}))$ that describes the transformation induced strainstrain interaction. The scaled form of the local free-energy $F_{L}$ is

$$
F_{L}=\int d \vec{r}\left[f_{l}(\epsilon(\vec{r}))+\frac{D}{2}(\nabla \epsilon(\vec{r}))^{2}-\sigma(\vec{r}) \epsilon(\vec{r})\right],
$$

where $\sigma$ is the scaled stress and $D$ is the scaled co-efficient of the inhomogeneous term. In the above equation, $f_{l}(\epsilon(\vec{r}))$ is the Landau polynomial for the first-order transformation given by

$$
f_{l}(\epsilon(\vec{r}))=\frac{\tau}{2} \epsilon(\vec{r})^{2}-\epsilon(\vec{r})^{4}+\frac{1}{2} \epsilon(\vec{r})^{6} .
$$

Here, $\tau=\left(T-T_{c}\right) /\left(T_{0}-T_{c}\right)$ is the scaled temperature. $T_{0}$ is the first-order transition temperature at which the free energy for the product and parent phases are equal, and $T_{c}$ is the temperature below which there are only two degenerate global minima, $\epsilon= \pm \epsilon_{M}$. It is well known that nucleation occurs at localized defect sites in the crystal such as dislocations and grain boundaries. ${ }^{40}$ This is modeled by a stress field ${ }^{28,30,41}$ represented by the third term in Eq. (2). This modifies the free energy $f_{l}$ in such a way that the austenite phase is locally unstable leading to the nucleation of the product phase.

In principle, long-range interaction between the transformed domains results from the elimination of the shear and bulk strains. In our model, this term has been introduced in a phenomenological way even though one can use the kernel obtained from St. Venant's principle. ${ }^{34,42}$ This has been taken to have the form

$$
F_{L R}\{\epsilon\}=-\frac{1}{2} \iint d \vec{r} d \vec{r}^{\prime} G\left(\vec{r}-\vec{r}^{\prime}\right) \epsilon^{2}(\vec{r}) \epsilon^{2}\left(\vec{r}^{\prime}\right) .
$$

We note here that unlike the long-range term emerging from the compatibility constraint which is linear in $\epsilon$, we have used $\epsilon^{2}$ term based on symmetry of the free energy under $\epsilon \rightarrow-\epsilon$. However, we mention here that we have checked that using the kernel obtained by using the compatibility condition gives results similar to the kernel used here. The kernel $G\left(\vec{r}-\vec{r}^{\prime}\right)$ is best defined in the Fourier representation as

$$
F_{L R}\{\epsilon\}=\frac{1}{2} \int d \vec{k} B\left(\frac{\vec{k}}{k}\right)\left\{\epsilon^{2}(\vec{r})\right\}_{k}\left\{\epsilon^{2}(\vec{r})\right\}_{k^{*}},
$$

where $\left\{\epsilon^{2}(\vec{r})\right\}_{k}$ is the Fourier transform of $\epsilon^{2}(\vec{r})$. The quantity $\left\{\epsilon^{2}(\vec{r})\right\}_{k^{*}}$ is the complex conjugate of $\left\{\epsilon^{2}(\vec{r})\right\}_{k}$. The kernel $B(\vec{k} / k)$ we use is designed to pick up the correct habit plane directions which in the present case are [11] and [1] $]$, which are the favorable directions of growth of the product phase. In addition, the free energy barrier is higher along the [10] and [01] directions. This is captured by the simple kernel given by

$$
B\left(\frac{\vec{k}}{k}\right)=-\beta \theta(k-\Lambda) \hat{k}_{x}^{2} \hat{k}_{y}^{2}
$$

where $\hat{k}_{x}$ and $\hat{k}_{y}$ are unit vectors in the $x$ and $y$ directions. The step function $\theta(k-\Lambda)$ has been introduced to impose a cutoff on the range of the long-range interactions. The constant $\beta$ is the strength of the interaction. The real-space picture of $B(\vec{k} / k)$ is similar to the long-range interaction of Kartha et $a .^{34}$ 
Following Bales and Gooding, ${ }^{36}$ we have used the Rayleigh dissipation functional ${ }^{43}$ to account for the dissipation accompanying the rapid interface motion given by

$$
R=\frac{1}{2} \gamma \int d \vec{r}\left(\frac{\partial}{\partial t} \epsilon(\vec{r}, t)\right)^{2} .
$$

It is well known that acoustic emission always accompanies the martensitic transformation and the physical cause is due to the abrupt motion of the interface. ${ }^{31}$ In our earlier papers, ${ }^{29,30}$ we have suggested that the above term can be interpreted as the energy dissipated in the form of acoustic signals during martensitic transformation. This interpretation is based on the well-known fact that acoustic emission accompanies abrupt motion of dislocations where the energy of AE signals is proportional to $\dot{\epsilon}^{2}$. Borrowing from this situation, and accounting for spatial inhomogeneity of the interfaces, we can write the energy dissipated to be proportional to $\int \dot{\epsilon}^{2} d \vec{r}$ which is identical to Eq. (7). Indeed, this dissipation term has been successfully used to account for several features of acoustic energy during the martensitic transformation such as the power-law statistics of the $\mathrm{AE}$ signals during thermal cycling and correlated acoustic emission when the system is cycled in a small temperature range. ${ }^{28-30}$

The Lagrangian then is given by $L=T-F$, where $F$ is the total free energy and $T$ is the kinetic energy associated with the system is given by

$$
T=\int d \vec{r} \rho\left[\left(\frac{\partial u_{x}(\vec{r}, t)}{\partial t}\right)^{2}+\left(\frac{\partial u_{y}(\vec{r}, t)}{\partial t}\right)^{2}\right] .
$$

Here $\rho$ is the mass density. We use this for obtaining the equations of motion for $\epsilon$.

\section{B. Magnetic free energy}

As stated earlier, one of our main interests is to study the direction of magnetic order in the martensite variants and in the parent phase. This is done by using a four-state clock model instead of continuous spin [U(1) asymmetry] systems as the latter does not support long-range order in two dimensions. The model used here describes anisotropic systems like Ni-Mn-Ga where there is a high degree of anisotropy and the easy axis of magnetization is along the short axis in the martensite phase. ${ }^{1,2,4}$ The free energy has contributions from exchange interaction, Zeeman energy, and magnetocrystalline anisotropy energy and is given by

$$
\begin{aligned}
f_{m}(\theta(\vec{r}))= & -\frac{1}{2} \sum_{\vec{r}^{\prime}} j_{p}(\epsilon(\vec{r})) \cos \left[\theta(\vec{r})-\theta\left(\vec{r}^{\prime}\right)\right]-\frac{k_{1}}{2} \cos [2 \theta(\vec{r}) \\
& -\pi / 4]-h \cos [\theta(\vec{r})-\phi],
\end{aligned}
$$

where the first term is the exchange energy with the sum taken over near neighbors, the second is the magnetic anisotropic energy, and the third, the Zeeman term. The spin vector $\theta$ is allowed to assume four values i.e., 0, 90, 180, and $270^{\circ}$ when located in the austenite phase, and four similar values with respect to the diagonal when it is in the martensite phase (see below). Moreover, the coefficient $j_{p}$ is chosen depending on whether the location of the spin $(p=a, m)$ is in the austenite or the martensite phase (in either of the two variants). When it is in the austenite phase, $\epsilon=0$, we use $j_{p}(\epsilon(\vec{r}))=j_{a}$ independent of the $x$ or $y$ direction. In the case of $\mathrm{Ni}_{2} \mathrm{MnGa},{ }^{1,4}$ it is known that the crystalline anisotropic direction coincides with the magnetic anisotropic direction which is the short $c$ axis. Thus, at a phenomenological level, we mimic this anisotropy of the martensite phase ( $\epsilon$ finite), by considering the magnitudes of the interaction along the short and long axis to be different, namely, $j_{p}(\epsilon(\vec{r}))=j_{m}$ $=j_{m s h}$ along the short axis and $j_{m}=j_{m l o}$ along the long axis of the martensite variant. (We note here that this choice can be adopted even if the $a$ axis is the easy axis of magnetization.) In addition, there is an on-site anisotropy term [the second term in Eq. (9)] with $k_{1}$ as the anisotropy coefficient. The anisotropy term is usually modeled by $\sin ^{2} \theta$, where $\theta$ is the angle measured with respect to the $x$ axis. From our earlier studies, ${ }^{28-30}$ we know that the martensitic variants are along the diagonal. Thus in order to have magnetic order parameter oriented along the easy directions in the martensite phase, we have subtracted $45^{\circ}$. This gives up to an additive term $\sin 2 \theta$. ( $h$ is the strength of the magnetic field and $\phi$ is the angle between the magnetic field and the $x$ axis.) Last, there is magnetostriction energy given by

$$
F_{m e}(\epsilon(\vec{r}), \theta(\vec{r}))=-\int d \vec{r} \frac{b_{1}}{\sqrt{2}} \epsilon(\vec{r}) \sin (2 \theta(\vec{r})),
$$

where $b_{1}$ is the phenomenological magnetoelastic constant. The effect of the strain produced during the martensitic transformation on the magnetic transition is captured by this term. Here again, considering the fact that $\epsilon$ takes on positive and negative values for the two twin variants and that these twins are oriented along the diagonal, the above term is chosen to be minimum along the diagonal. The total magnetic contribution to the free energy is given by the sum of $F_{m}(\theta(\vec{r}))\left[=\int f_{m}(\theta(\vec{r})) d \vec{r}\right]$ and $F_{m e}$.

The total free energy is minimized with respect to both the order parameters $\epsilon$ and $\theta$. Here, we note that while strain $\mathrm{OP}$ is continuous, the magnetic OP is discrete. Moreover, in our model, the martensitic transformation is considered athermal and is therefore described by deterministic equations obtained using

$$
\frac{d}{d t}\left(\frac{\delta L}{\delta \dot{u}_{i}}\right)-\frac{\delta L}{\delta u_{i}}=-\frac{\delta R}{\delta \dot{u}_{i}}, \quad i=x, y .
$$

In terms of rescaled space and time variables, we get

$$
\begin{aligned}
\frac{\partial^{2}}{\partial t^{2}} \epsilon(\vec{r}, t)= & \nabla^{2}\left[\frac{\partial f(\vec{r}, t)}{\partial \epsilon(\vec{r}, t)}-\sigma(\vec{r})-\nabla^{2} \epsilon(\vec{r}, t)+\gamma \frac{\partial}{\partial t} \epsilon(\vec{r}, t)\right. \\
& +2 \epsilon(\vec{r}, t) \int d \vec{k} B(\vec{k} / k)\left\{\epsilon^{2}(\vec{k}, t)\right\}_{k} e^{i \vec{k} \cdot \vec{r}} \\
& \left.-\frac{b_{1}}{\sqrt{2}} \sin (2 \theta(\vec{r}))\right] .
\end{aligned}
$$

Here, $\beta, \gamma$, and $b_{1}$ are scaled parameters. However, as the magnetic order parameter is allowed only four values (Ising like), it is most conveniently dealt with using the Metropolis Monte Carlo algorithm. 


\section{NUMERICAL SIMULATIONS}

Numerical simulations to study the evolution of strain morphology and the magnetic ordering are described below. We have solved Eq. (12) using Euler's scheme with periodic boundary conditions after discretizing it on a $N \times N$ grid. The grid size of the mesh used is $\Delta x=1$ and the smallest time step is $\Delta t=0.002$. Simulations were carried out for $N=64,128$, and 256 with a view to study finite-size effects. However, most of the results reported here are for $N=128$ except when reporting the influence of system size where we have shown $N=256$. A pseudospectral technique is employed to compute the long-range term. ${ }^{30}$ The cutoff $\Lambda$ in the long-range expression is chosen to be 0.1 . The inhomogeneous stress field $\sigma(\vec{r})$ is appropriately chosen to describe the multidefect configuration given by

$$
\sigma(\vec{r})=\sum_{j}^{j_{\max }} \sigma_{0}\left(\vec{r}_{j}\right) \exp \left(\frac{-\left|\vec{r}-\vec{r}_{j}\right|^{2}}{\zeta_{j}^{2}}\right),
$$

where $\sigma_{0}\left(\vec{r}_{j}\right)$ is the magnitude of the stress field at sites $\vec{r}_{j}$ which are randomly chosen defect sites, $j_{\text {max }}$ is the total number of defect sites, and $\zeta_{j}$ the width of the field. A random distribution of $0.1 \%$ defects is used which for $N=128$ gives $j_{\text {max }}=16$. The values of $\sigma_{0}\left(\vec{r}_{j}\right)$ are taken to be uniformly distributed in the interval $[-0.3,0.3]$. The parameters chosen for the martensite transformations are $\beta=30$ and $\gamma=1$. We start our simulations with the system being in a homogeneous state with $\epsilon(\vec{r}, 0)$ distributed in the interval [-0.005, 0.005].

On the discrete lattice, $\theta(\vec{r})=\theta_{i j}$, the initial values of the spin $\theta_{i j}$ at the location $i j$ are taken to be randomly oriented among $0,90,180$, or $270^{\circ}$ directions in the austenite phase and $45,135,225$, or $315^{\circ}$ in the martensite phase. Thus the allowed spin flips are through $\pm 90^{\circ}$. However, when crossing either the twin or the martensite-austenite boundaries, we allow spin flips of $\pm 45^{\circ}$ to ensure that the spin orientations across the boundaries change appropriately. The anisotropy coefficient is taken to be $k_{1}=0.6$ and the magnetostriction coefficient $b_{1}=0.6$ in our simulations (unless otherwise stated). The exchange interactions $j_{p}$ that fixes the Curie temperatures of the magnetic phases are chosen so as to obtain the required ordering of the Curie temperatures. In the Monte Carlo (MC) simulations, while computing the equilibrium magnetization at each temperature, starting from an initial disordered state, the first $2.0 \times 10^{5} \mathrm{MC}$ steps were discarded for thermalizing, and the averages are computed by sampling over configuration obtained in the next $1.0 \times 10^{5} \mathrm{MC}$ steps. (We find that this duration is adequate for obtaining steadystate values of the magnetization. We also note that as there is no quenched disorder, averaging over the initial set of configurations is not necessary.) The variance is calculated for each temperature by sampling uncorrelated configurations. ${ }^{44}$ In the martensite phase, we have assumed that $j_{m}$ is different along the short and long axis of the variant denoted by $j_{m s h}$ and $j_{m l o}$, respectively, as stated above.

As the magnetomartensite involves two coupled order parameters, simultaneous updating of both the order parameters becomes necessary. To see the possible kinds of simulations that approximate the desired properties mentioned in the in- troduction, we examine time duration over which the order parameters (OPs) reach near steady-state values, even as there is no mapping of MC time with real time. When athermal martensites are quenched from the austenite phase, it is well known that the value of the strain order parameter stabilizes rapidly at times scales determined by the velocity of sound. This should be compared with the time duration over which the total magnetization reaches a near steady-state value consistent with the temperature when quenched from a disordered state. Relevant to this is the fact that in our model, the magnetic order parameter is modeled using Ising-like spins. In this case, first domains of a specific magnetic order are formed followed by their growth. As the formation of domains and their subsequent coarsening is controlled by thermal fluctuations (in contrast, in athermal martensites, thermal fluctuations play little role in the kinetics of strain $^{30,31,34}$ ), the time duration to reach a near steady-state value of the total magnetization is expected to be much slower than the time required for strain to stabilize. Thus while studying the magnetization curves as well as visualizing the corresponding magnetic ordering in the two phases, as we are essentially dealing with a stationary situation, it is reasonable to assume that the time evolution of the system can be approximated by assuming that the strain order parameter reaches its steady state much faster than the magnetic order parameter. Thus in the first type of simulation, strain is evolved until a stationary morphology of the martensites is obtained and then a MC evolution of the magnetic order parameter is effected (or vice versa depending on the ordering of the transition and transformation temperatures). However, it is clear that this kind of simulation takes into account the influence of the already formed strain on magnetization or vice versa. Thus this type of simulation can only be regarded as a first approximation, as we shall see. To approximate the real situation where there is continuous feedback between the two order parameters, two more types of simulations are undertaken. A second type of simulation involves the evolution of strain OP for long enough time to reach stationary values followed by $\mathrm{MC}$ evolution required for stabilization of the magnetization ( $~ 300000$ steps). This sequence is repeated until steady-state values of both the OPs are achieved. Finally, we have also carried out a third type of simulation where in both order parameters are not allowed to reach a steady-state value during one cycle. Here, a cycle consisting of the strain OP developed for a short time during which the strain does not reach a stationary value followed by development of magnetic order for a short duration of MC steps, typically $50000 \mathrm{MC}$ steps. The simulations are repeated several cycles until both OPs stabilize.

\section{RESULTS}

Before proceeding further, we note that it is necessary to make a correspondence between the temperature scale used in the MC simulations with the temperature scale used in Eq. (12). In our MC simulations, the scale of temperature is in the range of unity as we choose $j_{a}$ in the neighborhood of unity (in units of $k_{B}$ ) for the austenite phase. However, the scaled temperature $\tau$ for the strain variable ranges 


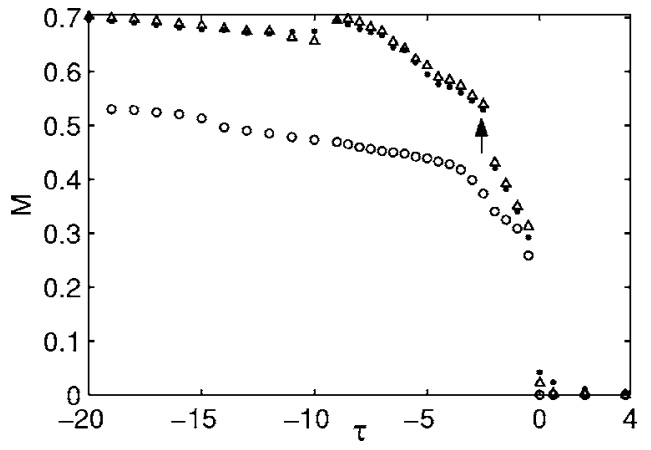

FIG. 1. Magnetization vs temperature plot for the condition $T_{c}^{A}<T_{m s}<T_{c}^{M}$. Curves denoted by $\bigcirc$ correspond to $N=128$ and $\triangle$ to $N=256$ when $T_{c}^{A}$ is close to $T_{M s}$. Curves denoted by $\bigcirc$ are for $N=128$ when $T_{c}^{A}$ is well below $T_{M s}$.

mostly over negative values. To map, we define a relation between the scaled temperature in MC simulations, $\tau_{M C}$, and $\tau$ such that $\tau=0$ maps to $\tau_{M C}=1$, i.e., $\tau_{M C}$ $=\left[\tau-\left(\tau_{M s}-\tau_{M f}\right)\right] /\left(\tau_{M f}-\tau_{M s}\right)$, where $\tau_{M C}=T / T_{c}$ with $T_{c}$ taken to be unity for a hypothetical austenite phase with $j_{a}=0.885$. $\left(\tau_{M s}\right.$ and $\tau_{M f}$ are the scaled martensite start and finish temperature, and the value of $\tau_{M s}-\tau_{M f}$ is determined numerically.) In experiments on FSMA, a small magnetic field is always used ${ }^{46,47}$ and the magnetization along the direction is measured. Following this, we have also used a small magnetic field along the positive $x$ direction. The magnetization is measured along this direction. (Moreover, we note that in general, there is no specified direction for the easy axis in the mixed austenite and martensite phase.) The results reported here are for periodic boundary conditions. The results for open boundary conditions are very similar except that the magnetization curves for this case lie marginally lower. For this reason, we shall only mention the results for one case (see case B). We now consider a few cases of the ordering of temperatures listed earlier.

\section{A. Case $T_{c}^{A}<T_{M s}<T_{c}^{M}$}

To obtain the above ordering of temperatures, we choose the exchange interactions in the austenite phase to be $j_{a}=0.826$, and for the martensite phase $j_{m s h}=0.95$ and $j_{m l o}=0.35$ along the easy and hard directions of the martensite variants, respectively. ${ }^{45}$ With the above value of $j_{a}$, we find $T_{c}^{A}=0.937$ which corresponds to $\tau=-1.98$. The above choice of $j_{m s h}$ and $j_{m l o}$ gives $T_{c}^{M}=1.0312$ (higher than $T_{M s}$, i.e., $\tau \sim 0.0$ ), which can only be determined numerically as it is influenced by $b_{1}$ and $k_{1}$. In this case, the Curie temperature of the martensite phase is not detectable as $T_{c}^{M}$ is higher than $T_{M s}$ and the high temperature austenite phase is paramagnetic. We have deliberately taken the values of $T_{c}^{M}, T_{M s}$, and $T_{c}^{M}$ to be close to obtain some features that will be not seen when they are well separated as we shall see. As the temperature is lowered below $T_{M s}(\tau \sim 0.0)$, some regions of the austenite phase undergo structural transformation to the martensite phase which then orders ferromagnetically. This is reflected as an abrupt increase in the magnetization around $\tau=0.0$ evident from Fig. 1 shown for two different system sizes $(N=256, \triangle$ and $N=128, \bigcirc)$. We note here that the effect of increasing the system size is that the magnetization curves of higher $N$ are sharper as it should be. The error bars on the magnetization are small, of the size of the symbol (and hence not shown), typically in the range 0.0001-0.002, being the largest near the transition temperature. As we decrease the temperature further, magnetization grows in proportion to the area fraction of the martensite phase until $T_{c}^{A}$ is reached. At this point $(\tau \approx-2.0)$, one again finds a sudden increase in the magnetization (arrow shown in Fig. 1 for the curve with $N=256, \triangle$ and $N=128, \bigcirc)$. This can be attributed to ordering of the untransformed fraction of the austenite phase. In addition, we note that the contribution to the total magnetization from the austenite phase is higher as magnetization (per unit area) of this phase is oriented along the $x$ direction compared to the martensite phase which is oriented along $\pm 45^{\circ}$. However, as we decrease the temperature, the decreasing volume fraction of the austenite compensates the oriented contribution from the austenite phase to the total magnetization. Consequently, a further decrease in temperature leads to a marginal decrease in the magnetization around $\tau=-10.0$ eventually saturating to a value around 0.7 . (Note that as $N$ increases, the magnitudes of the jumps at $\tau=-2.0$ and -10.0 also increase.) The sudden jump in the magnetization at $\tau=-2.0$ would not be seen if the value of $T_{c}^{A}$ is such that the volume fraction of the untransformed austenite phase is small. In such a case, the magnetization curve increases smoothly without such sharp changes. This is shown on the same figure ( $\bigcirc$ for $N=128$ in Fig. 1 ) when $T_{c}^{A}=0.765$ which corresponds to $\tau=-7.52$. For this case, the saturation value is much lower than for the case shown by the symbols and $\triangle$ as the parameter values are slightly different $\left(j_{a}=0.55\right.$, $j_{m s h}=0.90, j_{m l o}=0.35$, and $\left.k_{1}=0.5\right)$. On visual inspection, we find a large number of smaller sized twins and consequently, the interface occupies an appreciable area fraction of the transformed phase that is disordered leading to smaller magnetization.

Figure 2 shows a morphological plot of the magnetic ordering in the martensite domains at $\tau=-2.0$ for $N=128(\mathbf{O})$, the top curve shown in Fig. 1. Note that the austenite phase is disordered. It is also clear from Fig. 2 that the two different variants of the martensite phase have different orientations corresponding to the two easy directions of magnetization. Figure 3 shows the morphology for a lower temperature $(\tau=-9.0)$, a value at which the system has almost fully transformed to the martensite phase. Note that disorder is at the interface while both the twin variants are reasonably well ordered even though the twin domains are rather small.

$$
\text { B. Case } T_{c}^{M}<T_{M s}<T_{c}^{A}
$$

As the temperature is lowered below $T_{c}^{A}$, one first finds magnetic ordering of the austenite phase followed by the formation of the martensitic phase below $T_{M s}$ and then magnetic ordering of the martensite phase. Choosing $j_{a}=1.22$, one can estimate $T_{c}^{A}$ to be 1.38 which corresponds to $\tau_{c}^{A}$ $=12.28$. However, in our simulations, the transition temperature is identified by the peak in the susceptibility which in this case is seen at $\tau_{c}^{A}=12.8$. (The simulated $\tau_{c}^{A}$ is affected by 


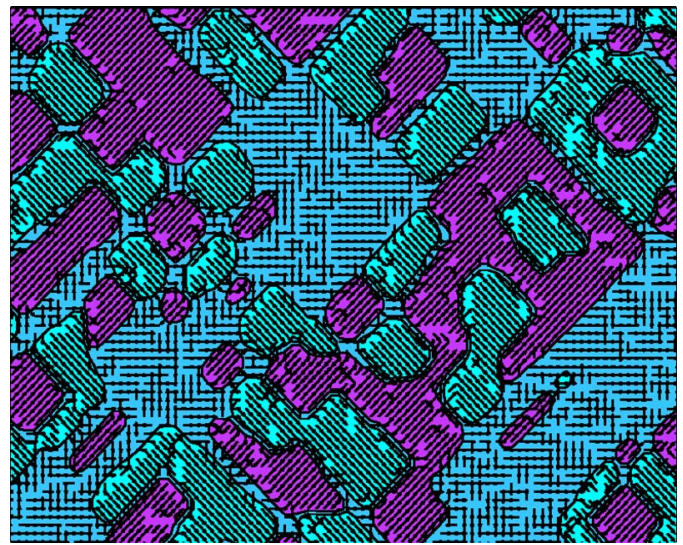

FIG. 2. (Color online) Morphology of the system at $\tau=-2.0$ for the case $T_{c}^{M}>\tau>T_{c}^{A}$. Here dark gray and light gray correspond to the two variants of the martensite and blue (intermediate gray) to the austenite phase. The magnetic ordering is present only in the martensite variants. For the sake of visual clarity a small region of $85 \times 55$ grid points is displayed.

finite sample size as well as small but finite magnetic field $h=0.025$ used to mimic the measurement of magnetization in FSMA.) The martensite transition temperature obtained numerically is $T_{c}^{M}=0.719$ for $j_{m s h}=0.7$ and $j_{m l o}=0.3$ which corresponds to $\tau_{M}^{c}=-9.0 .{ }^{45}$ The simulations have been carried out for three system sizes stated earlier.

The magnetization curves for this case are shown in Fig. 4 for two different sizes $N=128(\bullet)$ and $N=256(\bigcirc)$. In our simulations, we find a small amount magnetization at $\tau=12.8$. On cooling further, one observes a rapid increase in the magnetization $M$. From Fig. 4 , it is clear that the austenite phase reaches a near saturation value at $\tau \approx 0\left(\approx T_{M s}\right)$. On cooling below $T_{M s}$, the martensite phase formed is not magnetically ordered at this temperature and hence there is a sudden drop in the magnetization. Indeed, the extent of the drop is equal to the area fraction of the austenite phase that transforms at $\tau=0$. On further cooling below the Curie temperature of the martensite $T_{c}^{M}$, the magnetization again increases reaching a value of 0.55 as we approach $\tau=-20$. In

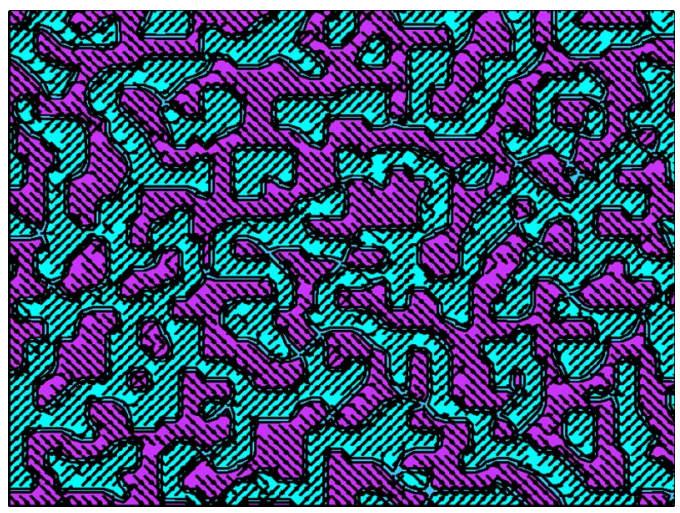

FIG. 3. (Color online) Morphology at $\tau=-9.0$ for the case $T_{c}^{M}>\tau>T_{c}^{A}$. The system is fully transformed to martensite, and the spins are well ordered. A small region of $85 \times 55$ grid points is shown. Here dark gray and light gray correspond to the two variants of martensites.

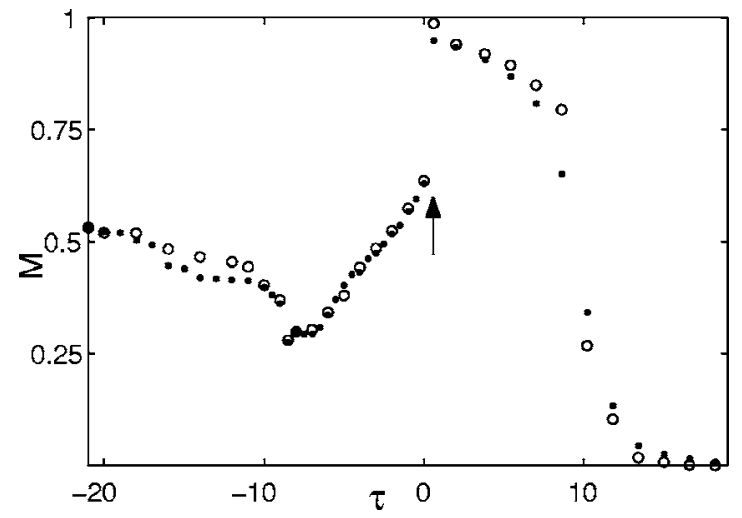

FIG. 4. Magnetization vs temperature plot for $T_{c}^{M}<T_{M s}<T_{c}^{A}$ for $N=128(\bigcirc)$ and $N=256(\bigcirc)$.

general the influence of increasing the system size is that the magnetization curve corresponding to higher $N$ [for instance, $N=256(\bigcirc)]$ is smoother than that for lower one $[N=128(\bullet)]$, particularly in the two phase region below $\tau=-20.0$. One can also see that the magnetization curve for $N=256$ is sharper compared to that for $N=128(\bullet)$ as we approach $T_{c}^{A}$ as expected. The error bars in the magnetization are small and are typically of the order of the symbol and hence not shown. Near $T_{c}^{A}$, the error is $\sim 0.006$.

Briefly, we mention here the effect of open boundary conditions for this case. We find that the values of magnetization are nearly the same as for the periodic case, but slightly lower, typically 0.01 to 0.02 lower than the corresponding curve with periodic boundary condition, except at $T_{M s}$ (or $\tau=0$ ), the point shown by the arrow lies lower by as much as 0.07 .

A snapshot of the spin ordering for $\tau=-3.0$ is shown in Fig. 5. It is clear that spins in the austenite phase are oriented along the $x$ axis while those in the martensite phase are disordered. We note that the maximum value of the magnetization in the transformed martensite phase (corresponding to

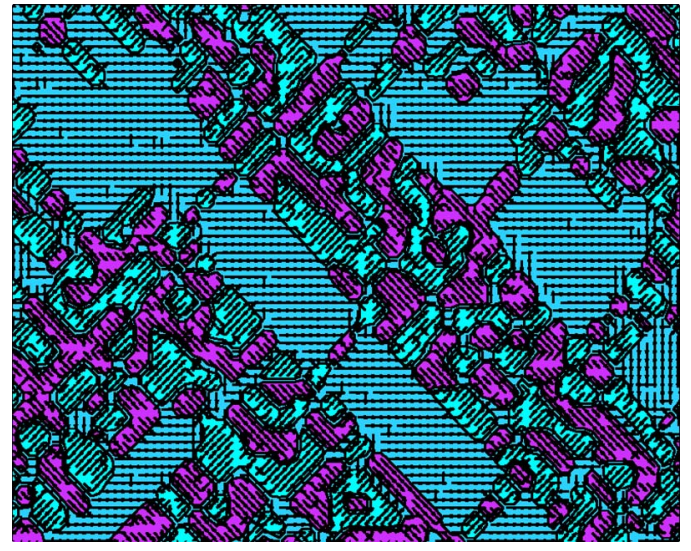

FIG. 5. (Color online) Ferromagnetic ordering of the austenite phase for the case $T_{c}^{M}<T_{M s}<T_{c}^{A}$ at $\tau=-3$. Note that the spins in the austenite phase which is intermediate gray are ordered and those in the martensite phase which are dark gray and light gray, corresponding to the two variants, are disordered. A small region of $85 \times 55$ grid points is shown. 


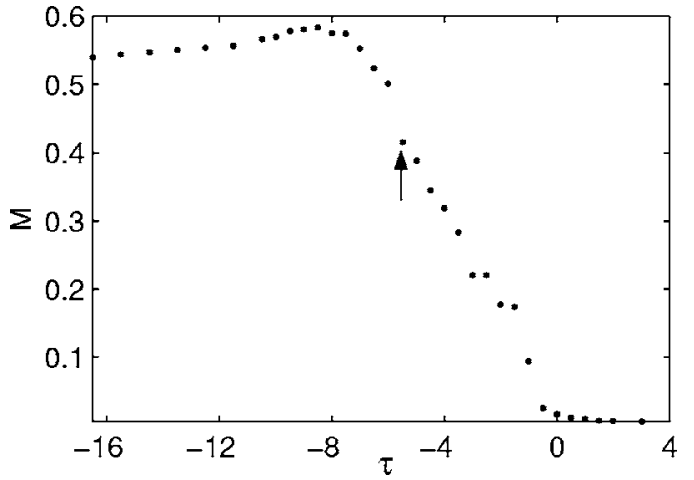

FIG. 6. Magnetization vs temperature plot for $T_{c}^{A}<T_{c}^{M}<T_{M s}$ for $N=128$.

$\tau=-20$ ) does not reach the saturation value $1 / \sqrt{2}$. This can be attributed to the fact that a substantial area fraction of the transformed phase is at the interface and hence is disordered. At these temperatures even as the martensite domains are quite fragmented, spins in the two martensite variants are ordered along their easy axis of magnetization, i.e., $\pm 45^{\circ}$. The spin ordering here is similar to $T_{c}^{M}<T_{M s}<T_{c}^{A}$ at similar temperature range, Fig. 3.

$$
\text { C. Case } T_{c}^{A}<T_{c}^{M}<T_{M s}
$$

To obtain the required ordering of the transition and transformation temperatures, we choose the exchange interaction $j_{a}=0.75$ for the austenite, and $j_{m s h}=0.9, j_{m l o}=0.65$ for the easy and hard directions of the martensite phase. With this choice, we get $T_{c}^{A}=0.83(\tau=-5.5)$ and $T_{c}^{M}=0.98(\tau=-0.64)$ for the austenite and the martensite phases, respectively. Above $T_{c}^{M}$, there is no ordering. As the temperature is lowered below $T_{c}^{M}$, the magnetization increases until $T_{c}^{A}$ $(\tau=-5.5)$ at which one sees a small jump in the magnetization arising from the fraction of the untransformed austenite phase as shown in Fig. 6. Thereafter, the magnetization increases to a maximum of 0.6 and falls marginally. This decrease can be attributed to decreasing fraction of the austenite phase. (The influence of increasing system size is similar to the earlier cases and hence not shown.)

\section{Influence of magnetization on strain}

It is evident from Eq. (12) that strain is affected by magnetization and vice versa when the system is in the martensite phase. This is a direct result of the magnetostriction coupling. However, the results of the previous section do not account for the mutual influence of the OPs as only one cycle of strain (or magnetization) followed by magnetization (strain) was taken into consideration. To observe the mutual influence of the OPs, several such cycles have to be allowed to reach the steady state. We shall illustrate this by considering the case $T_{c}^{A}<T_{M s}<T_{c}^{M}$. As the high-temperature phase is the paramagnetic austenite phase, we first obtain the initial stationary state for the strain order parameter by quenching the system below $T_{M S}$ (i.e., $\tau$ just less than 0.0 ). This is followed by allowing the magnetic order parameter to reach a stationary value consistent with the strain value. (Near sta-

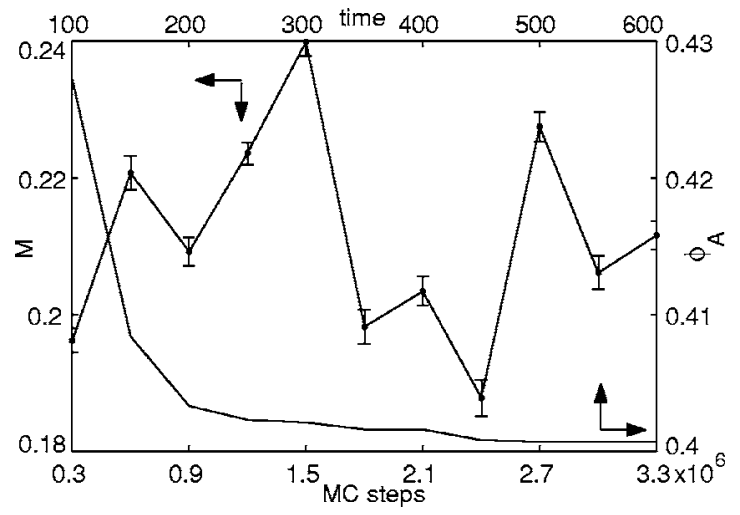

FIG. 7. Plot of magnetization and area fraction vs $\mathrm{MC}$ steps and time for $T_{c}^{A}<T_{M s}<T_{c}^{M}$ at $\tau=-0.5$ for $N=128$.

tionary $\phi_{A}$ values are reached in 30 time units.) Each magnetization run was for $300000 \mathrm{MC}$ steps and $M$ is calculated over the last $100000 \mathrm{MC}$ steps. This process is repeated for several cycles until stationary values of the OPs are obtained. We find that the maximum change in the OPs occurs in the first few cycles. While the magnetization which goes through oscillatory changes takes a much longer time to stabilize, the strain decreases monotonically. A plot of the strain and magnetization (along with the error bars) as a function of time and MC steps is shown in Fig. 7. Snapshots of the strain morphology at the end of the first and last cycle are shown in Fig. 8. It is evident that the martensite boundaries have changed significantly from the initial configuration.

We have carried out a third type of simulation wherein strain and magnetization are allowed to develop for a short time so that they do not reach their steady-state values. Considering the fact that there is no correspondence between MC time and real time, and in real systems, there is a continuous feedback between the two order parameters during time evolution of the system, simulations where the OPs are allowed to evolve for short durations are closer to continuous time development of both the OPs. Another interpretation that is possible is that this can approximate a situation where the time scales of both the order parameters are comparable. For this case, starting from the austenite phase, we quench the system below $T_{M s}$ (i.e., $\tau$ just less than 0.0 ) and the strain is allowed to develop for a short time (here $t=20$ units). Starting from this initial martensite phase, we allow the magnetic order to develop for a short time (MC steps are typically 50000 steps). This is repeated for several cycles. Both the strain and magnetic order parameters ( $M$ averaged over $30000 \mathrm{MC}$ steps) are monitored at the end of each cycle. (The error bars are considerably larger as the number of MC steps used is smaller.) We find that while the maximum change occurs in both the OPs during the first cycle, the magnetic order takes longer to reach a steady value going through oscillatory changes. The stationary values of the OPs are obtained after about 12 cycles. This is shown in Fig. 9. Comparing Fig. 9 with Fig. 7, it is clear that the eventual near steady-state values of both strain and magnetization values are nearly the same. In fact, even the final strain configuration corresponding to the last cycle in Fig. 9 is very similar to the right configuration shown in Fig. 8. The percentage 

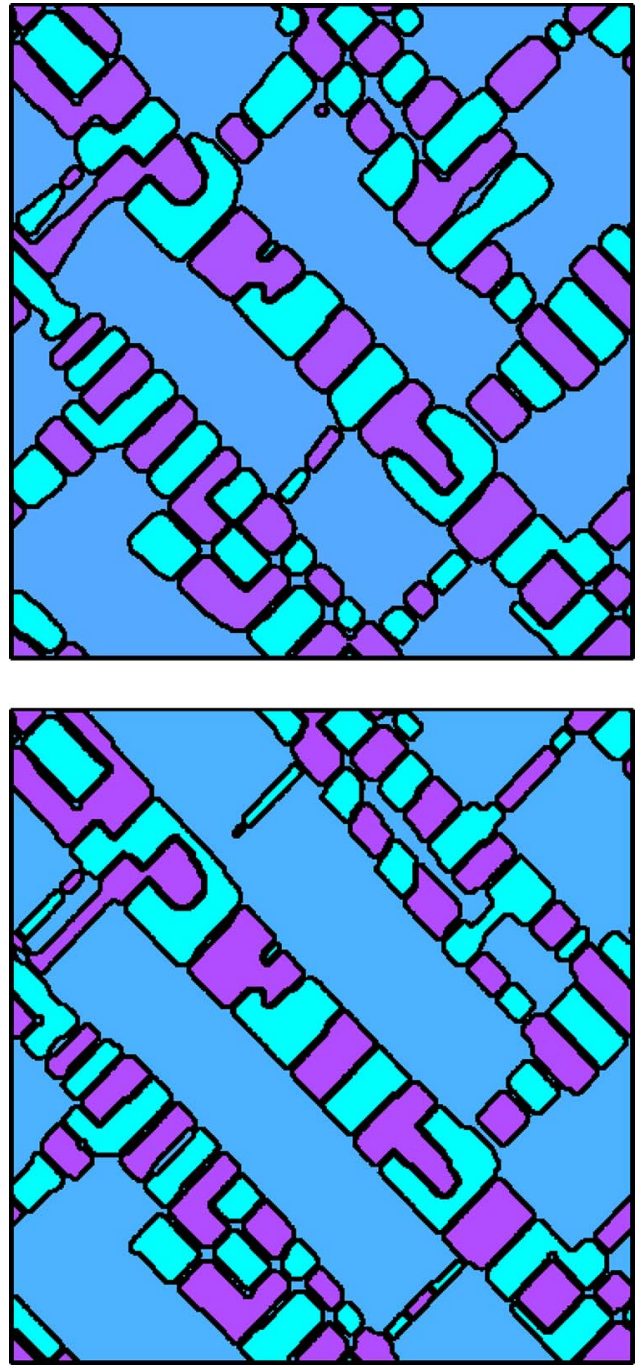

FIG. 8. (Color online) Strain morphology of the system for $T_{c}^{A}<T_{M s}<T_{c}^{M}$ at $\tau=-0.5$ before the start (top) of the cycles and at the end configuration (bottom). The change in the area fraction of the martensite phase due to the magnetization is clearly seen. The entire $128 \times 128$ grid of points is shown. Dark gray and light gray are the martensite variants and intermediate gray is the austenite phase.

change at the end of the simulation in strain OP with respect to the initial value is $\sim 6.0 \%$ in both these simulations. In contrast, the magnetic order changes by almost $\sim 10 \%$ with respect to the intial value in the first type of simulation and is even more in the second type of simulation.

As the maximum change in strain occurs in the first cycle, we have examined the influence of temperature on this differential change. This is shown in Fig. 10 for two conditions $T_{c}^{A}<T_{M s}<T_{c}^{M}(\bigcirc)$ and $T_{c}^{A}<T_{c}^{M}<T_{M s}(\diamond)$. As can be seen, in both cases, the differential change is maximum at the onset of the martensite phase.

\section{E. Hysteresis}

We have also studied the hysteresis loop keeping the temperature at an appropriate value. Here the field is changed at

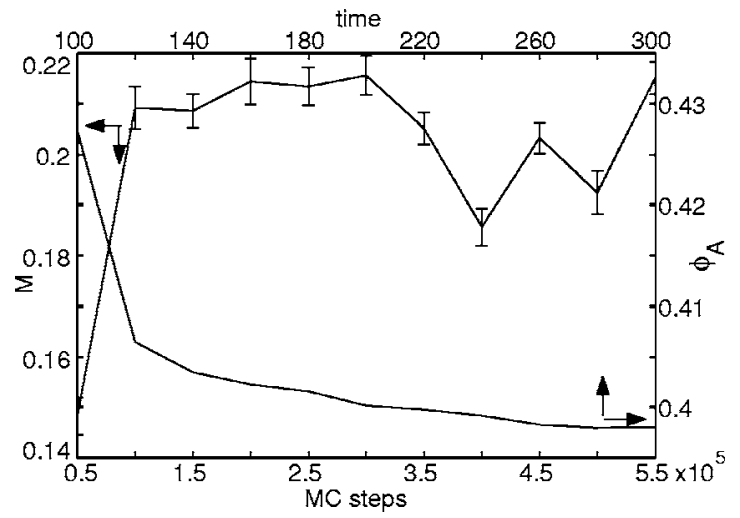

FIG. 9. Plot of magnetization and area fraction vs MC steps and time for $T_{c}^{A}<T_{M s}<T_{c}^{M}$ at $\tau=-0.5$ for $N=128$.

a specified rate from zero value to a positive maximum (corresponding to the saturation value of the magnetization) and then reversing the field to the negative maximum, and back again to the positive maximum completing a cycle. First, the system is thermalized to the desired temperature, here, $\tau=-3.0$, for the case $T_{c}^{A}<T_{M s}<T_{c}^{M}$. The loop is swept by varying the field at intervals of $h=0.05$, except near saturation where it is varied at intervals of 0.2 (beyond 0.6). Magnetization is evolved for $500 \mathrm{MC}$ steps at each value of the field. At this temperature, $44 \%$ of the system is transformed to the martensite phase which is ferromagnetic. Figure 11 shows the hysteresis cycle for this condition that is nearly symmetric.

As is clear from the previous section, magnetization influences strain. In the present case, even though the change in the strain configuration as $h$ is cycled is not detectable in the morphological plots, one can detect this change by monitoring the dissipation function $R(\dot{\epsilon})$ shown in Fig. 12. Further, as shown in our earlier work, ${ }^{28-30}$ strain changes occur by the jerky movement (stick-slip-like movement) of the interface even as they are small in this case. Indeed, $R(\dot{\epsilon})$ shows a sequence of bursts. Due to the symmetry of the hysteresis cycle, the dissipation functions $R(\dot{\epsilon})$ for the forward and the reverse cycle are nearly the same as can be seen from Fig. 12. We find that $R(\dot{\epsilon})$ is highest when the field is close to zero. Here, it is pertinent to recall that in our earlier work we have shown that the dissipation, i.e., $R(\dot{\epsilon})$, accom-

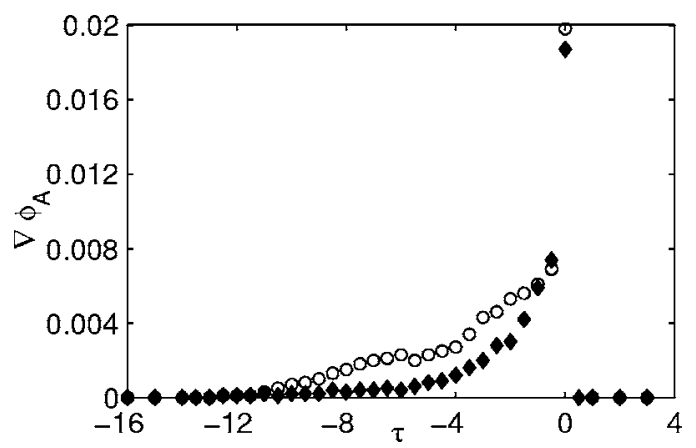

FIG. 10. Difference in area fraction vs scaled temperature, before and after magnetization for the cases $T_{c}^{A}<T_{M s}<T_{c}^{M}(\bigcirc)$ and $T_{c}^{A}<T_{c}^{M}<T_{M s}(\diamond)$ for $N=128$. 


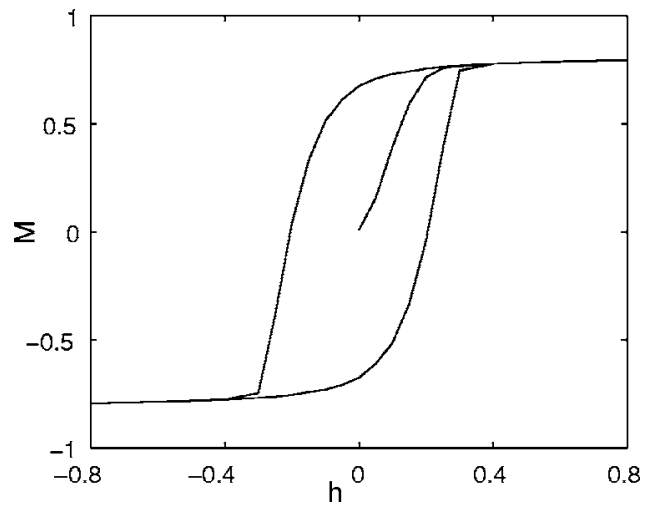

FIG. 11. Hysteresis plot for the case $T_{c}^{A}<T_{M s}<T_{c}^{M}$ at $\tau=-3.0$ and $N=128$.

panying the strain changes have all the characteristic features of acoustic emission. ${ }^{28-30}$ If this interpretation is followed here, the dissipation is similar to the Barkhausen noise observed during the hysteresis. Even though these actually correspond to acoustic waves, the driving force for the movement of the martensite interface comes from the changes in the magnetization.
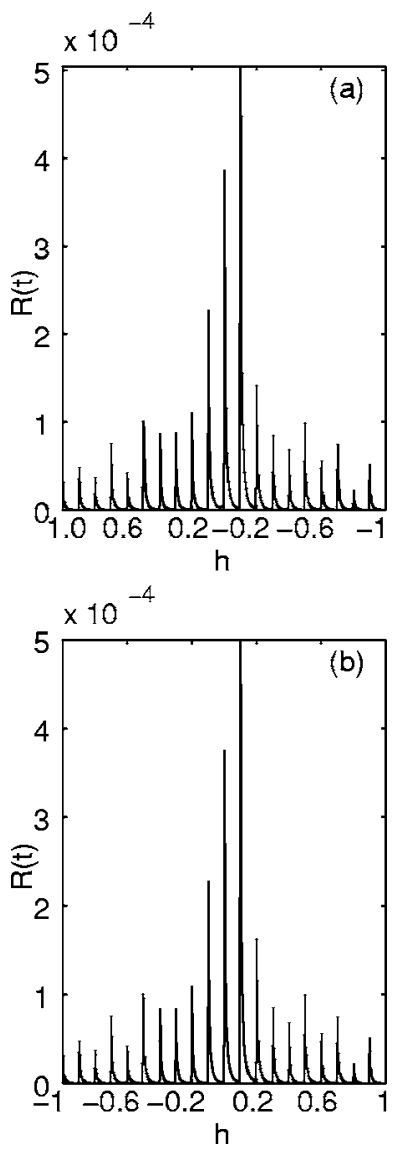

FIG. 12. Dissipation plotted as a function of external applied field for the case $T_{c}^{A}<T_{M s}<T_{c}^{M}$ at $\tau=-3.0$ and $N=128$. (a) Reverse cycle (from $h=1.0$ to $h=-1.0$ through zero field), (b) forward cycle (from $h=-1.0$ to 1.0 ).

\section{SUMMARY AND CONCLUSIONS}

In summary, we have extended the recently introduced two-dimensional square-to-rectangle martensite model to include the magnetic order parameter through a four-state clock model to study ferromagnetic martensites. The model includes all the features of the structural transformation (i.e., long-range strain interaction, inertia, and defect centers that act as nucleation sites) in addition to magnetic transition (which includes exchange interaction and anisotropy) and mutual coupling through a magnetostriction term. By choosing appropriate exchange interactions in the two phases, the model is used to explain the magnetization curves of the austenite-martensite system under various ordering of the Curie temperatures and the martensite transformation temperature. These simulations refer to the steady-state condition wherein only one cycle of strain and magnetization is monitored (the initial state could be an ordered magnetic state followed by strain or vice versa depending on the ordering of the temperatures), and the subsequent influence of magnetization on strain (or vice versa) has been ignored. Even at this level one sees that there is a subtle interplay between the growth of the strain and magnetic order parameters that leads to a jump in magnetization at $T_{c}^{A}$ in cases when the $T_{c}^{A}$ of the austenite phase is lower than and close to $T_{c}^{M}$ (or $T_{M s}$ ), a feature not predicted by the mean-field models. Our simulations also show that the orientations of the magnetic order of the $\pm \epsilon$ twins are along $\pm 45^{\circ}$ directions, whereas it is orientated along the $x$ axis in the austenite phase. Further, the model also captures the abrupt change in the orientation of the magnetization across the two variants of the martensite as also the austenite-martensite phase boundary.

Two more types of simulation have been carried out to monitor the mutual influence of the OPs which show that the strain order parameter is influenced by the magnetic order and vice versa in a significant way. In particular, the magnetic order influences the strain morphology of the martensite domains in a substantial way. It is interesting to note that while strain OP decreases monotonically, the magnetization changes in an oscillatory way. This is clearly due to the invariance of the magnetostriction coupling being linear in strain and quadratic in $M$. As this mutual influence is triggered by the magnetostriction coupling, and considering large strains induced in Terfenol-D are due to magnetostriction, the model may have some relevance to Terfenol-D. Studies on hysteresis show that dissipation $R(\dot{\epsilon})$ exhibits a sequence of bursts whose magnitudes reach a maximum as the applied field passes through zero value. Taking the interpretation that $R(\dot{\epsilon})$ mimics acoustic signals suggested in our earlier work, ${ }^{28-30}$ this feature is similar to the Barkhausen noise. While the origin of the Barkhausen noise is attributed to pinning and depinning of the magnetic domains, in our case, the strain changes result from magnetization which in turn act as a driving force causing the jerky movement of the austenite-martensite interface. ${ }^{28-30}$ Finally, it should be possible to adopt this approach to systems where ferroelectric order parameter has strong coupling to strain.

Several points of general nature in modeling magnetomartensites need clarification. First, it is well known that the 
mixed martensite-austenite phase is metastable in nature which is captured by the long-range interaction term between the transformed domains that generates a self-blocking mechanism (see Refs. 28-30). Here, it is worth noting that though the long-range interaction between the transformed phases has been introduced here in a phenomenological way, one can actually use the long-range kernel obtained by eliminating the bulk dilational and shear strain components by demanding the compatibility condition. ${ }^{34}$ Indeed, we have carried out simulations using such a long-range kernel replacing the present one. We find that most results reported here remain unchanged ${ }^{48}$ Second, the process of twinning in the model is of dynamical origin, being the result of a dynamic balance between the kinetic energy and dissipation. ${ }^{36}$ As a result, the process of twinning and the transformation, both proceed at the time scale of sound velocity. Thus, within the scope of the model, kinetic effects are neglegible. As the twin boundaries have a finite width, though small, this area is magnetically disordered. More importantly, as neighboring martensite variants are perpendicular to each other coupled with the fact that the short axis is the easy axis of magnetization, we find only $90^{\circ}$ Bloch walls within the scope of a four-state clock model. For the same reason, the model cannot support a $180^{\circ}$ Bloch wall which needs spins that can be continuously rotated. However, within the scope of twodimensional martensite model, it is nontrivial to incorporate a continuous spin even in the presence of strong anisotropy.

\section{ACKNOWLEDGMENT}

We wish to thank Professor S. Bhaskaran for suggesting the four-state clock model to deal with the magnetic order parameter.
*Electronic address: garani@mrc.iisc.ernet.in

${ }^{1}$ A. Sozinov, P. Yakovenko, and K. Ullakko, Mater. Sci. Forum 373-376, 35 (2001).

${ }^{2}$ T. Sakamoto, T. Fukuda, T. Kakeshita, T. Takeuchi, and K. Kishio, J. Appl. Phys. 93, 8647 (2003).

${ }^{3}$ L. Straka and O. Heczko, J. Appl. Phys. 93, 8636 (2003).

${ }^{4}$ A. DeSimone and R. D. James, J. Mech. Phys. Solids 50, 283 (2002).

${ }^{5}$ R. Tickle and R. D. James, J. Magn. Magn. Mater. 195, 627 (1999).

${ }^{6}$ K. Ullakko, J. K. Huang, C. Kantner, R. C. O'Handley, and V. V. Kokorin, Appl. Phys. Lett. 69, 1966 (1996).

${ }^{7}$ A. A. Likhachev and K. Ullakko, Phys. Lett. A 275, 142 (2000).

${ }^{8}$ T. Yamamoto, M. Taya, Y. Sutou, Y. Liang, T. Wada, and L. Sorensen, Acta Mater. 52, 5083 (2004).

${ }^{9}$ R. C. O’Handley, J. Appl. Phys. 83, 3263 (1998).

${ }^{10}$ R. C. O'Handley, S. J. Murray, M. Marioni, H. Nembach, and S. M. Allen, J. Appl. Phys. 87, 4712 (2000).

${ }^{11}$ S. J. Murray, M. Marioni, S. M. Allen, R. C. O'Handley, and T. A. Lograsso, Appl. Phys. Lett. 77, 886 (2000).

${ }^{12}$ H. D. Chopra, C. Ji, and V. V. Kokorin, Phys. Rev. B 61, R14913 (2000).

${ }^{13}$ A. Steuwer, T. Mori, H. Kato, and T. Wada, J. Appl. Phys. 94, 2761 (2003).

${ }^{14}$ T. Wada, Y. Liang, H. Kato, T. Tagawa, M. Taya, and T. Mori, Mater. Sci. Eng., A 361, 75 (2003).

${ }^{15}$ Y. Murakami, D. Shindo, M. Suzuki, M. Ohtsuka, and K. Itagaki, Acta Mater. 51, 485 (2003).

${ }^{16}$ E. C. Oliver, T. Mori, M. R. Daymond, and P. J. Withers, Acta Mater. 51, 6453 (2003).

${ }^{17}$ O. Heczko, K. Jurek, and K. Ullakko, J. Magn. Magn. Mater. 226-230, 996 (2001).

${ }^{18}$ J. Cui, T. W. Shield, and R. D. James, Acta Mater. 52, 35 (2004).

${ }^{19}$ V. A. Chernenko, C. Segui, E. Cesari, J. Pons, and V. V. Kokorin, Phys. Rev. B 57, 2659 (1998).

${ }^{20}$ A. N. Vasil'ev, A. D. Bozhko, V. V. Khovailo, I. E. Dikshtein, V. G. Shavrov, V. D. Buchelnikov, M. Matsumoto, S. Suzuki, T. Takagi, and J. Tani, Phys. Rev. B 59, 1113 (1999).
${ }^{21}$ V. A. Chernenko, V. A. L'vov, S. P. Zagorodnyuk, and T. Takagi, Phys. Rev. B 67, 064407 (2003).

${ }^{22}$ R. D. James and M. Wuttig, Philos. Mag. A 77, 1273 (1998).

${ }^{23}$ D. I. Paul, J. Marquiss, and D. Quattrochi, J. Appl. Phys. 93, 4561 (2003).

${ }^{24}$ V. D. Buchel'nikov, A. T. Zayak, A. N. Vasil'ev, D. L. Dalidovich, V. G. Shavrov, T. Takagi, and V. V. Khovailo, Zh. Eksp. Teor. Fiz. 119, 1166 (2001) [JETP 92, 1010 (2001)].

${ }^{25}$ V. D. Buchel'nikov, A. N. Vasil'ev, A. T. Zayak, and P. Entel, Zh. Eksp. Teor. Fiz. 119, 1176 (2001) [JETP 92, 1019 (2001)].

${ }^{26}$ V. D. Buchel'nikov, V. S. Romanov, and A. T. Zayak, J. Magn. Magn. Mater. 191, 203 (1999).

${ }^{27}$ A. N. Vasil'ev, V. D. Buchel'nikov, T. Takagi, V. V. Khovailo, and E. I. Estrin, Phys. Usp. 46, 559 (2003).

${ }^{28}$ R. Ahluwalia and G. Ananthakrishna, Phys. Rev. Lett. 86, 4076 (2001), and references therein.

${ }^{29}$ S. Sreekala and G. Ananthakrishna, Phys. Rev. Lett. 90, 135501 (2003).

${ }^{30}$ S. Sreekala, R. Ahluwalia, and G. Ananthakrishna, Phys. Rev. B 70, 224105 (2004).

${ }^{31}$ E. Vives, J. Ortín, L. Mañosa, I. Ràfols, R. Pérez-Magrané, and A. Planes, Phys. Rev. Lett. 72, 1694 (1994); E. Vives, I. Ràfols, L. Mañosa, J. Ortín, and A. Planes, Phys. Rev. B 52, 12644 (1995); Ll. Carrillo, Ll. Mañosa, J. Ortín, A. Planes, and E. Vives, Phys. Rev. Lett. 81, 1889 (1998); F. C. Lovey and V. Torra, Prog. Mater. Sci. 44, 189 (1999).

${ }^{32}$ I. S. Muto, S. Takeda, R. Oshima, and F. E. Fujita, J. Phys.: Condens. Matter 1, 9971 (1989).

${ }^{33}$ I. M. Robertson and C. M. Wayman, Philos. Mag. A 48, 421 (1983); 48, 443 (1983); 48, 629 (1983).

${ }^{34}$ S. Kartha, J. A. Krumhansl, J. P. Sethna, and L. K. Wickham, Phys. Rev. B 52, 803 (1995).

${ }^{35}$ R. Oshima, M. Sugiyama, and F. E. Fujita, Metall. Trans. A 19A, 803 (1988).

${ }^{36}$ G. S. Bales and R. J. Gooding, Phys. Rev. Lett. 67, 3412 (1991).

${ }^{37}$ E. Obradó, E. Vives, and A. Planes, Phys. Rev. B 59, 13901 (1999).

${ }^{38}$ M. Rao, H. R. Krishnamurthy, and R. Pandit, Phys. Rev. B 42, 
856 (1990).

${ }^{39}$ J. Garcia-Otero, M. Porto, J. Rivas, and A. Bunde, J. Magn. Magn. Mater. 203, 268 (1999).

${ }^{40}$ P. L. Ferraglio and K. Mukherjee, Acta Metall. 22, 835 (1974).

${ }^{41}$ Y. Wang and A. G. Khachaturyan, Acta Mater. 49, 1165 (2001); Y. M. Jin, A. Artemev, and A. G. Khachaturyan, ibid. 49, 2301 (2001); A. Artemev, Y. Wang, and A. G. Khachaturyan, ibid. 48, 2503 (2000).

${ }^{42}$ T. Lookman, S. R. Shenoy, K. Ø. Rasmussen, A. Saxena, and A. R. Bishop, Phys. Rev. B 67, 024114 (2003).

${ }^{43}$ L. D. Landau and E. M. Lifschifz, Theory of Elasticity, 3rd ed. (Pergamon, Oxford, 1986).

${ }^{44}$ K. Binder and D. W. Heerman, Monte Carlo Simulation in Statistical Physics: An Introduction (Springer-Verlag, Berlin, 1988).
${ }^{45}$ We stress that these unrealistic values of $j_{m s h}, j_{m l o}$, and $j_{a}$ are necessary to have a good separation between $T_{c}^{A}$ and $T_{c}^{M}$. The anisotropy and the magnetoelastic constants taken to be $k_{1}$ $=0.5$ and $b_{1}=0.6$ are also unrealistic. These were, however, the minimum values of the parameters for which the martensitic domains of reasonably small twins sizes would get ordered along the easy direction in a reasonable time.

${ }^{46}$ V. A. Chernenko, V. A. L'vov, V. V. Khovailo, T. Takagi, T. Kanomata, T. Suzuki, and R. Kainuma, J. Phys.: Condens. Matter 16, 8345 (2004).

${ }^{47}$ T. Kakeshita, T. Fukuda, T. Terai, T. Takeuchi, and K. Kishio, J. Phys. IV 112, 93 (2003).

${ }^{48}$ S. Sreekala and G. Ananthakrishna (unpublished). 